\title{
Evaluation of ovarian reserve in terms of ovarian volume, antral follicule count and hormonal tests in Hashimoto's thyroiditis
}

Turkan Mete', Aysu Basak Ozbalci², Elif Kilic Kan'1, Gulhan Duman', Halise Cinar Yavuz ${ }^{3}$, Gultekin Ozan Kucuk ${ }^{4}$, Ismail Ozer ${ }^{5}$, Hacer Pinar Ozturk ${ }^{6}$, Aysegul Atmaca ${ }^{7}$

${ }^{1}$ Ministry of Health, Samsun Training and Research Hospital Department of Endocrinology and Metabolism, ${ }^{2}$ Ministry of Health, Samsun Training and Research Hospital Department Of Radiology, ${ }^{3}$ Turgut Ozal University, School of Medicine Department of Endocrinology and Metabolism, ${ }^{4}$ Ministry of Health, Samsun Training and Research Hospital Department Of General Surgery, ${ }^{5}$ Ministry of Health, Samsun Training and Research Hospital Department Of Nephrology, ${ }^{6}$ Ministry of Health, Samsun Training and Research Hospital Department Of Internal Medicine, ${ }^{7}$ School of Medicine, Ondokuz Mayis University Department of Endocrinology and Metabolism

\section{OBJECTIVES}

Ovarian reserve is very important in reproductive women for menstrual regularity and for pregnancy development. In women of reproductive age the prevalence of hypothyroidism ranges from $2 \%$ to $4 \%[1,2]$. In this age group, Hashimoto's thyroiditis (HT) is the most common cause of hypothyroidism [3]. Hypothyroidism is associated with a broad spectrum of reproductive disorders. Additionally, HT may be associated with autoimmune diseases that cause ovarian dysfunction like premature ovarian failure. Our aim is to investigate whether ovarian reserve is decreased in patients with euthyroid HT.

\section{METHODS}

Our study included 110 euthyroid HT patients and 72 age matched healthy women (controls; C). In all patients FSH, LH E2, and P levels were measured from blood samples, and ovarian volumes (OV) and antral follicule count (AFC) were measured using ultrasonography at 3rd day of menstruation.

\section{RESULTS}

No statistically significant difference was detected in FSH, LH, E2 and P levels between groups ( $p$ value respectively; $0.117,0.420$, $0.941,0.644)$. Total ovarian volumes were not statistically different between groups $(p=0.165)$. Total AFC were significantly lower in the HT group than the controls $(p<0.001)$. Total AFC were strongly and negatively correlated with AntiTPO ( $r=-0.215, p=0.004)$ and AntiTg ( $r=-0.251, p=0.001)$ (Table 1).
Table 1. Summary of the hormonal and ultrasonographic parameters of the study and control groups

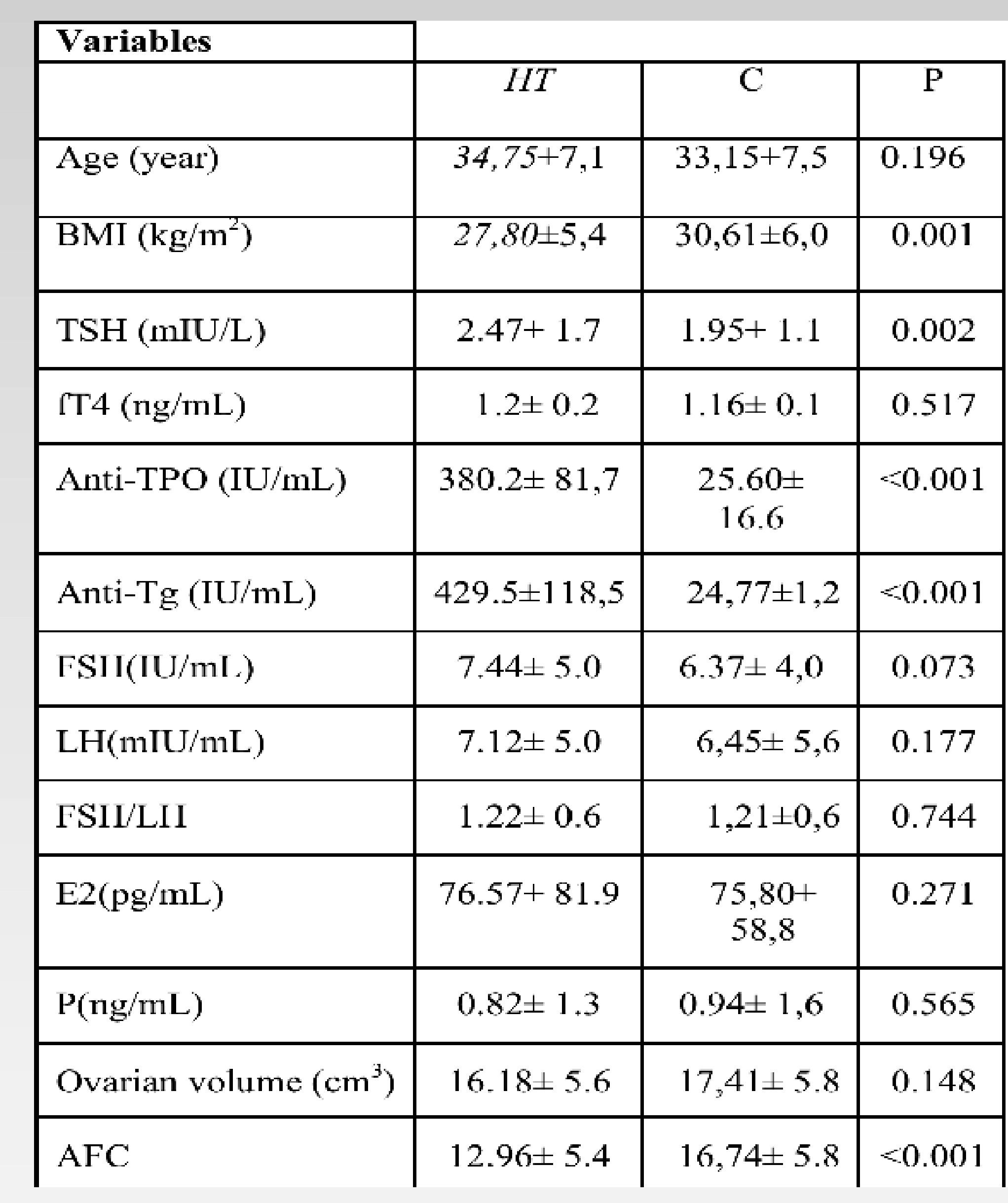

\section{CONCLUSIONS}

Our study showed that women with HT who are euthyroid have low antral follicle numbers than healthy controls although they have similar LH, FSH, E2, and P levels and ovarian volumes.

\section{REFERENCES:}

1. Poppe K, Velkeniers B, Glinoer B. The role of thyroid autoimmunity in fertility and pregnancy. Nat Clin Pract Endocrinol Metab. 2008; 4: 394-405.

2. Wang C, Crapo LM. The epidemiology of thyroid disease and implications for screening. Endocrinol Metab Clin North Am. 1997; 26: 189-218.

3. Pearce EN, Farwell AP, Braverman LE: Thyroiditis. N Engl J Med. 2003; 348: 2646-2655 